\title{
Efficacy of Sinemet CR4 in subgroups of patients with Parkinson's disease
}

\author{
S A FACTOR, $\dagger$ J R SANCHEZ-RAMOS, * W J WEINER,* ANGELA M INGENITO* \\ From The University of Miami, Department of Neurology, ${ }^{*}$ Miami, Florida, and Albany Medical College, \\ Department of Neurology, Albany, New York, USA
}

SUMmARY The efficacy of Sinemet CR4 (50/200) was compared to standard Sinemet (25/100) in an open label crossover study in 22 patients with Parkinson's disease. All patients experienced end of dose failure and 11 had dyskinesia. Unified Parkinson's disease, Hoehn and Yahr, Schwab and England scores, number of hours on per day, number of hours of dyskinesia per day, daily dose of levodopa, and number of doses per day were monitored at the end of each treatment period and the results compared. The only significant difference in these parameters between the CR4 and standard Sinemet treatment periods in the entire group was a decrease in hours of dyskinesia per day. Two subgroups of CR4 responders were specifically examined. The first subgroup was characterised by a significant increase in on time per day with CR4 therapy. These patients had an older age of onset of Parkinson's disease and a shorter duration of disease and fluctuations than the rest of the patients. The second subgroup was characterised by the presence of dyskinesia with standard Sinemet therapy and a significant decrease in hours of dyskinesia per day with CR4 therapy. Both subgroups required a significantly higher daily dose of levodopa while on CR4. It is concluded that CR4 may be useful in increasing hours on per day in subgroups of Parkinson's disease patients who have less severe fluctuations. It may also be useful in decreasing the number of hours of dyskinesia per day.

Levodopa in combination with a peripheral dopadecarboxylase inhibitor (carbidopa or benserazide) is the mainstay of therapy in Parkinson's disease. It significantly improves patient disability' and increases life span to near normal duration. ${ }^{2}$ However, after 5 years of therapy complications emerge in approximately one half of patients ${ }^{3}$ and after 10 years these complications are experienced in greater than $80 \% .^{4}$ The adverse effects include loss of efficacy, psychiatric side effects, and motor fluctuations. The most commonly recognised fluctuations include end of dose failure, peak dose dyskinesia, random on-off phenomenon, and ineffectiveness of individual doses. The mechanisms behind these fluctuations have not been fully elucidated, however, pharmacokinetic and pharmacodynamic factors, and the natural progression of disease appears to be involved. ${ }^{5}$ Pharmacokinetic factors, reflecting alterations in bioavailability of

Address for reprint requests: William J Weiner, MD. University of Miami, School of Medicine Department of Neurology (D4-5) P.O. Box 016960 Miami, Florida 33101, USA

Received 8 April 1988 and in revised form 31 July 1988. Accepted 8 August 1988 levodopa in the striatum, seem to be more important when planning therapeutic strategies for end of dose failure, peak dose dyskinesia, and ineffectiveness of individual doses. Although it is suggested that on-off phenomenon is primary related to pharmacodynamic factors (alteration in dopamine receptor number and sensitivity) even this fluctuation tends to occur during a drop in plasma levodopa levels. ${ }^{5}$ Many therapeutic strategies have been recommended in patients with motor fluctuations to maintain constant levodopa plasma levels and to improve its bioavailability. Most well known of these is to administer frequent, small doses of levodopa/carbidopa. Patients, however, find this plan inconvenient and of limited usefulness. In addition, this strategy may play a role in the formation of more unpredictable responses which occur as a result of longer duration therapy. ${ }^{67}$ Alterations in dietary protein levels, ${ }^{8}$ direct duodenal placement of levodopa with a nasogastric tube 9 and intravenous forms of levodop $\mathrm{a}^{610}$ have also been utilised with some success. Results of these and other studies suggest that the striatum will respond to steady levels of levodopa with smoother motor output. ${ }^{112}$ The problem remains how to improve the bioavailability of levodopa. 
Attempts at therapy with sustained release Sinemet formulations, Sinemet CR3, ${ }^{1314}$ have resulted in suboptimal responses.

We evaluated the efficacy and safety of Sinemet CR4. This formulation is a tablet made with a slowly erodable matrix (different from that used in Sinemet CR3) allowing for a prolonged, steady release of levodopa and carbidopa into the bowel. This results in more sustained levels of levodopa in the plasma which may lead to prolonged bioavailability of levodopa to the striatum and improved motor performance in Parkinson's disease patients.

\section{Patients and methods}

Thirty patients initially enrolled in the study. Twenty two, 13 male and nine female, patients completed it. Ages varied from 41 to 75 (mean 63.8) and duration of disease varied from 4 to 26 years (mean 10.6). All patients had end of dose failure, 13 had dyskinesia and three experienced on-off phenomenon prior to enrolment with a duration of fluctuations ranging from 1 to 10 years (mean 3.8). Peak dose dyskinesia and diphasic dyskinesia were not differentiated. All patients were receiving a minimum of four doses of levodopa/carbidopa per day. Other than levodopa, alldopamine receptor agonists were discontinued at least 14 days prior to enrolment. Amantadine and anticholinergics were permitted during the study. Neuroleptic and monoamine oxidase preparations were not permitted. Patients with active cardiovascular, hepatic, renal, haematologic, pulmonary or neoplastic disease were excluded.

The study was an open label comparison of standard Sinemet (25/100) and Sinemet CR4 (50/200). During the first treatment period (weeks 1 to 4 ) patients received Sinemet. If the patients had been taking $10 / 100$ or $25 / 250$ tablets prior to enrolment they were switched to the $25 / 100$ tablets at the start of the first treatment period. During this time an optimal dosage schedule was achieved. At the beginning of the second treatment period (week 5) therapy was changed to Sinemet CR4 using the following guidelines; the total daily dose of levodopa was 100 to $120 \%$ of that at the end of the first treatment period and the number of doses per day was decreased by 25 to $50 \%$. During weeks 5 to 8 the dosage schedule was optimised. Three patients required one extra week and two patients required two extra weeks to optimise
CR4 dosage. Patient visits were at baseline and at the end of weeks $1,2,4,5,6,8$ ( 9 and 10 if necessary). Severity of disease was measured at baseline, end of standard Sinemet therapy (week 4) and end of CR4 therapy (week 8,9 or 10) utilising the unified Parkinson's disease scale, Hoehn and Yahr scale ( $H$ and $Y$ ) and Schwab and England scale ( $S$ and $E$ ). In addition, each patient completed a diary recording hours on, on with dyskinesia, off, and sleep for 2 days of each week. Patients recorded their motor response the same 2 days of each week for the entire study. All adverse reactions were monitored.

For statistical evaluation the unified Parkinson's disease scale was separated into the activities of daily living score (ADL) which was recorded for both on and off time, and the motor exam score. The $\mathrm{S}$ and $\mathrm{E}$ scale was also scored for on and off time. These scores, the $H$ and $Y$ stage, number of hours on per day (this included hours on with and without dyskinesia) and numbers of hours with dyskinesia per day from the end of the standard Sinemet treatment period and the end of the Sinemet CR4 treatment period were compared using the paired $t$ test. In addition, total daily dose of levodopa and total number of doses per day at the end of each treatment period were compared.

\section{Results}

Eight patients withdrew from the study within the first 5 weeks (after one week or less of therapy with Sinemet CR4). Three because of psychiatric illness and therapy (one was hospitalised for depression and the other two required antidepressant therapy which excluded them from the study). Two other patients withdrew because of a worsening of Parkinson's disease with initiation of CR4. In these patients CR4 was either slow to take effect or had no effect at all. They were unwilling to attempt further therapy with higher doses of CR4 before dropping out. One patient had an allergic reaction to the standard Sinemet 25/100 which was apparently related to the yellow dye in the tablet. This had occurred with previous therapy and the reaction was generalised pruritis. Two others gave no particular reasons.

The mean scores for ADL(on), ADL(off), motor exam, $H$ and $Y$, and $S$ and $E$ (on and off), the number

Table 1 A comparison of 22 Parkinson's disease patients on standard Sinemet and Sinemet CR4

\begin{tabular}{|c|c|c|c|}
\hline Parameters & $\begin{array}{l}\text { Mean scores at the end of standard } \\
\text { Sinemet treatment period }\end{array}$ & $\begin{array}{l}\text { Mean scores at the end of CR4 } \\
\text { treatment period }\end{array}$ & Comparison \\
\hline $\begin{array}{l}\text { Motor exam } \\
\text { ADL (on) } \\
\text { ADL (off) } \\
\text { S and E (on) } \\
\text { S and E (off) } \\
\text { H and Y } \\
\text { Hours on per day } \\
\text { Hours dyskinesia per day } \\
\text { Total daily levodopa dose } \\
\text { Number of doses per day }\end{array}$ & $\begin{array}{l}18 \cdot 1 \\
8.8 \\
21.9 \\
81.4 \\
49 \cdot 1 \\
2 \cdot 5 \\
10.0 \\
2.4 \text { sem } 0.6 \\
913.6 \\
6.4\end{array}$ & $\begin{array}{c}16 \cdot 9 \\
9 \cdot 2 \\
22 \cdot 9 \\
82 \cdot 1 \\
48 \cdot 8 \\
2 \cdot 5 \\
10 \cdot 1 \\
1.4 \operatorname{sem} 0.4 \\
1245 \cdot 5 \\
5 \cdot 5\end{array}$ & $\begin{array}{l}p=N S \\
p=N S \\
p=N S \\
p=N S \\
p=N S \\
p=N S \\
p=N S \\
p<0.05 \\
p=N S \\
p=N S\end{array}$ \\
\hline
\end{tabular}

sem $=$ standard error of the mean

NS $=$ not significant 
Table 2 A comparison of 7 CR4 responders (subgroup 1), characterised by an increase in on time per day, with standard Sinemet and Sinemet CR4 therapy

\begin{tabular}{lccc}
\hline Parameters & $\begin{array}{l}\text { Mean scores at the end of standard } \\
\text { Sinemet treatment period }\end{array}$ & $\begin{array}{l}\text { Mean scores at the end of CR4 } \\
\text { treatment period }\end{array}$ & Comparison \\
\hline Motor exam & 22.9 & 18.4 & $\mathrm{p}=\mathrm{NS}$ \\
ADL (on) & 11.3 & 11.6 & $\mathrm{p}=\mathrm{NS}$ \\
ADL (off) & 26.9 & 26.1 & $\mathrm{p}=\mathrm{NS}$ \\
S and E (on) & 72.9 & 74.3 & $\mathrm{p}=\mathrm{NS}$ \\
S and E (off) & 38.6 & 42.9 & $\mathrm{p}=\mathrm{NS}$ \\
H and Y & 2.9 & 2.6 & $\mathrm{p}=\mathrm{NS}$ \\
Hours on per day & $6.9 \mathrm{sem} 0.7$ & $12.1 \mathrm{sem} 1.0$ & 0.05 \\
Hours dyskinesia per day & 4.0 & 1.7 & $\mathrm{p}=\mathrm{NS}$ \\
Total daily levodopa dose & $971 \mathrm{sem} 56.5$ & $1471 \mathrm{sem} 126.4$ & $\mathrm{p}<0.005$ \\
Number of doses per day & 5.6 & 5.4 & $\mathrm{p}=\mathrm{NS}$ \\
\hline
\end{tabular}

sem $=$ standard error of the mean

$\mathrm{NS}=$ not significant

of hours per day with dyskinesia, number of hours on per day, daily dose of levodopa and number of doses per day at the end of each treatment period are recorded in table 1 . In these 22 patients the only significant change was in hours of dyskinesia per day which decreased on CR4 $(p<0.05)$. Although only 11 patients in treatment period 1 and 10 in treatment period 2 experienced dyskinesia, all 22 patients were included in the statistical analysis because all were susceptible to the occurrence of this adverse effect. There was a trend towards increased daily dose of levodopa. The number of doses per day was the same for standard Sinemet and Sinemet CR4.

Seven patients could be evaluated as a separate subgroup (subgroup 1) because they had a 33 to $141 \%$ increase in hours on per day with CR4 as compared with standard Sinemet which was significant (table 2). Subgroup 1 was compared with the other 15 patients with regard to age of onset of Parkinson's disease,

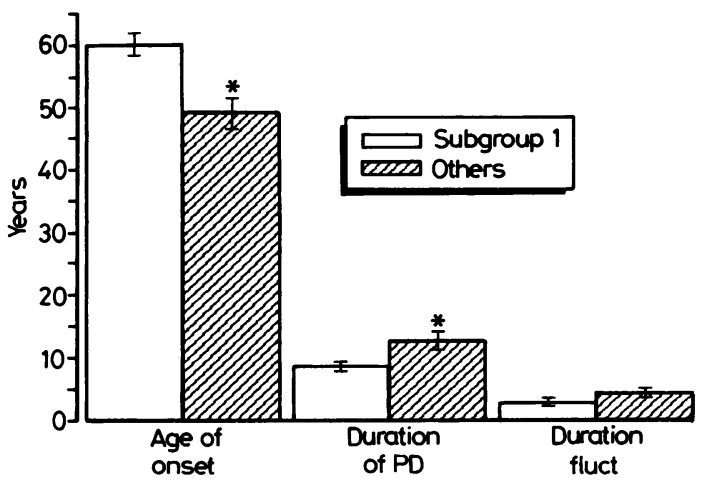

Fig 1 Seven CR4 responders (subgroup 1) are compared with the other 15 patients with regard to age of onset of Parkinson's disease, duration of disease, and duration of fluctuations. Subgroup 1 had a significantly older age of onset of Parkinson's disease (60.1 vs 49.0), a significantly shorter duration of disease ( 8.6 vs 12.5 years), and a trend towards a shorter duration of fluctuations ( 2.8 vs 4.3 years).

* denotes statistical significance. duration of Parkinson's disease and duration of fluctuations. These CR4 responders had a significantly older age of onset of Parkinson's disease ( $60 \cdot 1$ vs $49 \cdot 0)$ and significantly shorter duration of disease ( $8.6 \mathrm{vs}$ 12.5 years, $\mathrm{p}<0.05)$. A trend toward a shorter duration of fluctuations was also observed ( 2.8 vs 4.3 years) (fig 1). We also compared ADL(on), ADL(off), motor examination, $\mathrm{H}$ and $\mathrm{Y}, \mathrm{S}$ and $\mathrm{E}(\mathrm{on})$, $S$ and $E(o f f)$, hours of dyskinesia per day and total levodopa dose and number of doses per day in this subgroup for the end of each treatment period (table 2). The only significant change from standard Sinemet therapy to CR4 therapy (other than the increase in on time) was an increase in daily dose of levodopa which was $52 \%$ higher with CR4 therapy. Non-significant trends were observed in motor exam score which was lower with CR4 therapy and in hours of dyskinesia per day which was lower with CR4 therapy. Two patients with the most substantial decrease in hours of dyskinesia per day with CR4 were in this subgroup. None of the patients with on-off phenomenon were in this subgroup.

Eleven patients experienced dyskinesia at the end of the standard Sinemet treatment period. Of these, nine had a decrease in the number of hours of dyskinesia per day with CR4, two remained the same. None of the patients had more hours of dyskinesia with CR4 than standard Sinemet. Three patients with no hours of dyskinesia at the end of the standard Sinemet treatment period experienced mild dyskinesia in the CR4 period; however, this was eliminated by small manipulations of Sinemet CR4 doses resulting in no dyskinesia by the end of the treatment period. Of the nine patients with a decrease in the number of hours of dyskinesia with CR4 only three decreased by more than 1 hour. One patient decreased from 6 hours to 4 , one from 10 to 5 and the other from 9 hours to 0 .

Statistical comparison revealed that the decrease in hours of dyskinesia per day in the group of nine patients discussed above was significant (table 3 ) allowing for their evaluation as a separate subgroup 
Table 3 A comparison of 9 CR4 responders (subgroup 2), characterised by the presence of dyskinesia with standard Sinemet therapy improvement of dyskinesia with CR4, on standard Sinemet and Sinemet CR4

\begin{tabular}{|c|c|c|c|}
\hline Parameters & $\begin{array}{l}\text { Mean scores at the end of standard } \\
\text { Sinemet treatment period }\end{array}$ & $\begin{array}{l}\text { Mean scores at the end of CR4 } \\
\text { treatment period }\end{array}$ & Comparison \\
\hline $\begin{array}{l}\text { Motor exam } \\
\text { ADL (on) } \\
\text { ADL (off) } \\
\text { S and E (on) } \\
\text { S and E (off) } \\
\text { H and Y } \\
\text { Hours on per day } \\
\text { Hours dyskinesia per day } \\
\text { Total daily levodopa dose } \\
\text { Number of doses per day }\end{array}$ & $\begin{aligned} 14 \cdot 3 \\
7 \cdot 4 \\
21 \cdot 7 \\
81 \cdot 1 \\
43 \cdot 8 \\
2 \cdot 4 \\
8.9 \\
4.9 \operatorname{sem} 1 \cdot 01 \\
800 \text { sem } 90.9 \\
6.3\end{aligned}$ & $\begin{array}{c}13 \cdot 3 \\
8 \cdot 3 \\
22 \cdot 4 \\
80 \cdot 0 \\
41 \cdot 3 \\
2 \cdot 3 \\
11 \cdot 8 \\
2.4 \operatorname{sem} 0.62 \\
1089 \operatorname{sem} 159 \cdot 3 \\
5 \cdot 3\end{array}$ & $\begin{array}{l}p=N S \\
p=N S \\
p=N S \\
p=N S \\
p=N S \\
p=N S \\
p=N S \\
p<0.005 \\
p<0.005 \\
p=N S\end{array}$ \\
\hline
\end{tabular}

sem $=$ standard error of the mean

NS $=$ not significant

(subgroup 2). Subgroup 2 was compared with the other 13 patients with regard to age of onset and, duration of Parkinson's disease, and duration of fluctuations. This subgroup had a significantly younger age of onset of Parkinson's disease (47.6 vs 56) than the rest of the patients $(p<0.05)$. No difference was seen in duration of Parkinson's disease (11.1 vs 11.4 years). A trend towards a longer duration of fluctuations ( 4.8 vs 3.2 ) was observed (fig 2 ). We then compared mean values of all parameters at the end of the standard Sinemet period with those at the end of the CR4 period (table 3). In addition to hours of dyskinesia per day, the only other significant difference was in total daily levodopa dose which was $26 \%$ higher with CR4 therapy. There was also a nonsignificant trend towards an increase in on time per day with CR4.

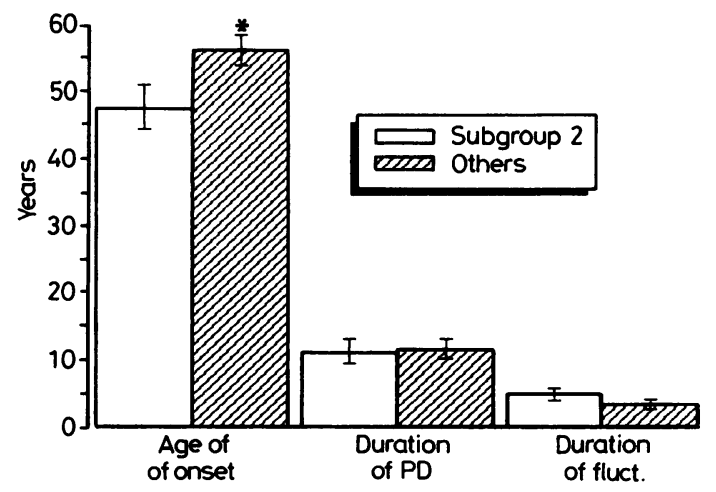

Fig 2 Nine CR4 responders (subgroup 2) are compared with the other 13 patients with regard to age of onset of Parkinson's disease, duration of disease, and duration of fluctuations. Subgroup 2 had a significantly younger age of onset of Parkinson's disease (47.6 vs 56.0), and a trend towards a longer duration of fluctuations ( $4 \cdot 8 \mathrm{vs} 3 \cdot 2$ years). Duration of disease was the same in both groups (11.1 vs 11.4 years).

*denotes statistical significance.
Three patients experienced unpredictable on-off effect prior to enrolment into this study. None of these patients improved with regard to on time. One patient also had dyskinesia which decreased by 1 hour per day on Sinemet CR4. Mean age of onset of Parkinson's disease in these three patients was $\mathbf{4 4 . 3}$ (range 35 to 50), mean duration of disease was 20 years (range 11 to 26) and mean duration of fluctuations was 5.6 years (range 2 to 10 ).

Adverse effects experienced while on CR4 were similar to that observed in patients treated with standard Sinemet. Two patients experienced hallucinations, two had light-headedness, one had nausea, and one had slight eosinophila.

\section{Discussion}

Other studies evaluating the efficacy of Sinemet CR4 in patients with Parkinson's disease have been reported. ${ }^{15-17}$ Goetz et al ${ }^{15}$ observed that 19 out of 20 patients responded to Sinemet CR4. Significant improvement in disability scores, $\mathrm{H}$ and $\mathrm{Y}$ stage, and hours on per day were observed. Cedarbaum et al $^{16}$ and Leibermann et $a l^{17}$ observed that all patients did not respond to CR4 which is in agreement with our findings. Cedarbaum $e a{ }^{16}$ observed an increase hours on per day and improvement in the ADL (on) score in 11 of 13 patients; however, no analysis of patient characteristics which might determine response was performed. Cedarbaum et al $^{16}$ and Goetz et al $^{15}$ observed the total daily dose of levodopa to be relatively unchanged in their patients. Despite the fact that this was true for our entire patient population, in the two subgroups of responders which we evaluated the total daily dose was significantly higher in the Sinemet CR4 treatment period. In addition, in the three previous reports of CR4 therapy ${ }^{15-17}$ fewer doses per day were required with CR4 therapy. We observed that the number of doses per day with CR4 was relatively unchanged as compared with standard Sinemet therapy. Finally, Cedarbaum et al ${ }^{16}$ observed an increase in dyskinesia 
in those patients already experiencing that adverse effect. They associated it with higher plasma trough levels of levodopa with CR4 as compared with standard Sinemet and claimed that this level increased with each dose to a point which resulted in increased afternoon dyskinesia. Goetz et al ${ }^{15}$ also observed this increase in trough levels of plasma levodopa and they observed that more patients experienced dyskinesia with CR4; however, the dyskinesia was less severe. Whether or not these trough levels play a role in the occurrence of dyskinesia in patients treated with CR4 remains to be elucidated. In our experience, patients tended to have less dyskinesia despite much higher doses of levodopa in the CR4 treatment period. As the results of other double blind and long term evaluations of Sinemet CR4 therapy become available, explanations regarding these differences between studies should emerge.

We compared the efficacy of Sinemet CR4 with standard Sinemet in 22 patients with Parkinson's disease and fluctuations in disability, most notably end of dose failure and dyskinesia. In this group only one parameter of evaluation was significantly different and that was hours of dyskinesia per day which was lower during CR4 therapy. Severity of disease as measured with the unified Parkinson's disease, $\mathrm{H}$ and $\mathrm{Y}$, and $S$ and $E$ scales as well as the number of hours of on time per day was unchanged. Because of the decrease in dyskinesia it is likely that the quality of on time was improved. The daily dosage of levodopa and number of doses per day during CR4 treatment was not significantly different from that observed with standard Sinemet treatment.

We observed that different subgroups of patients responded to CR4 in different ways. Subgroup 1 was characterised by a significant increase in hours on per day. These patients were older at the onset of Parkinson's disease and had a shorter duration of disease and fluctuations than the rest of the patients. Subgroup 2 was characterised by the presence of dyskinesia with standard Sinemet therapy and a significant decrease in hours per day of dyskinesia with Sinemet 'CR4. There were no changes in the number of hours on per day. These patients had a younger age of onset of Parkinson's disease and longer duration of fluctuations. Both subgroups required a significantly higher daily dose of levodopa with CR4 therapy to attain their respective responses.

Fluctuations in response to levodopa become more complex and less predictable with longer duration of disease and longer duration fluctuations. ${ }^{418}$ Fluctuations typically begin with end of dose failure. The duration of response becomes variable with time in end of dose failure leading to a less predictable response. With time on-off phenomenon becomes mixed with end of dose failure and in the later stages on-off effect predominates. Young onset Parkinson's disease patients tend to have earlier, more severe fluctuations than older onset patients. ${ }^{19}$ We suggest that response to Sinemet CR4 is in part dependent on age of onset of Parkinson's disease, duration of Parkinson's disease, and duration of fluctuations. Those patients with short duration of disease, short duration of fluctuations, and older age of onset of Parkinson's disease, all of which suggest more predictable, less severe fluctuations may tend to respond with an increase in on time per day and less dyskinesia. Those patients with longer duration, more severe fluctuations and dyskinesia and onset of Parkinson's disease at a younger age may respond to Sinemet CR4 with a decrease in dyskinesia without an increase in hours of on time. Those in which unpredictable response to standard Sinemet predominates CR4 may not be effective. A similar pattern of response was observed with intravenous levodopa therapy by Mouradian et al. ${ }^{18}$ This variation in response to CR4 depending on duration of disease and fluctations suggests that pharmacodynamic factors may play a more important role in motor fluctuations of long duration. In patients with early stage fluctuations CR4 may be useful by functionally correcting the loss of ability of the nigro-striatal dopaminergic system to buffer variations of plasma and striatal levodopa. This dysfunction may be due to the natural progression of Parkinson's disease. ${ }^{1820}$ We conclude that Sinemet CR4 may be useful in subgroups of patients characterised by shorter duration and less severe motor fluctuations.

This study was supported by NIH grant NS07238, Merck Sharp and Dohme, and the National Parkinson Foundation.

\section{References}

1 Markham $\mathrm{CH}$, Diamond SG. Long term follow-up of early dopa treatment in Parkinson's disease. Ann Neurol 1986;19:365-72.

2 Diamond SG, Markham CH, Hoehn MM, McDowell FH, Muenter MD. Multicenter study of Parkinson mortality with Early versus later dopa treatment. Ann Neurol 1987;22:8-12.

3 deJong GT, Meerwalt JD, Schmitz PIM. Factors that influence the occurrence of response variations in Parkinson's disease. Ann Neurol 1987;22:4-7.

4 Marsden CD, Parkes JD, Quinn N. Fluctuations of disability in Parkinson's disease: clinical aspects (Chapter 7). In: Marsden CD and Fahn S, eds. Movement Disorders. London: Butterworth Scientific, 1982:96-122.

5 Fahn S. Fluctuations of disability in Parkinson's disease: pathophysiological aspects (Chapter 8). In: Marsden 
CD and Fahn S, eds. Movement Disorders. London: Butterworth Scientific, 1982:123-45.

6 Nutt JG, Woodward WR. Levodopa pharmacokinetics and pharmacodynamics in fluctuating parkinsonian patients. Neurology 1986;36:739-44.

7 Nutt JG. On-Off phenomenon: relation to levodopa pharmacokinetics and pharmacodynamics. Ann Neurol 1987;22:535-40.

8 Pincus JH, Barry KB. Influence of dietary protein on motor fluctuations in Parkinson's disease. Arch Neurol 1987;44:270-2.

9 Kurlan R, Rubin AJ, Miller C, Rivera-Calimlin L, Clarke A, Shoulson I. Duodenal delivery of levodopa for onoff fluctuations in parkinsonism: preliminary observations. Ann Neurol 1986;20:262-5.

10 Nutt JG, Woodward WR, Hammerstad JP, Carter JH, Anderson JL. The "on-off" phenomenon in Parkinson's disease: relation to levodopa absorption and transport. $N$ Engl J Med 1984;310:483-8.

11 Yahr MD. Limitations of long term use of antiparkinsonian drugs. Can J Neurol Sci 1984;11:191-4.

12 Obeso JA, Luquin MR, Martinez Lage JM. Intravenous Lisuride corrects oscillations of motor performance. Ann Neurol 1986;19:31-35.

13 Nutt JG, Woodward WR, Carter JH. Clinical and biochemical studies with controlled release levodopa/ carbidopa. Neurology 1986;36:1206-11.
14 Cedarbaum JM, Breck L, Kutt H, McDowell FH. Controlled release levodopa/carbidopa. I. Sinemet CR3 treatment of response fluctuations in Parkinson's disease. Neurology 1987;37:233-41.

15 Goetz CG, Tanner CM, Klawans HL, Shannon KM, Carroll VS. Parkinson's disease and motor fluctuations: Long acting carbidopa/levodopa (CR-4-Sinemet). Neurology 1987;37:875-8.

16 Cedarbaum JM, Breck L, Kutt H, McDowell FH. Controlled release levodopa/carbidopa. II. Sinemet CR4 treatment of response fluctuations in Parkinson's disease. Neurology 1987;37:1607-12.

17 Leiberman AN, Miller E, Gopinathan G, Neophytides A. A comparison of controlled-release Sinemet $50 / 200 \mathrm{mg}$ with standard Sinemet $25 / 100 \mathrm{mg}$ in Parkinson's disease. Ann Neurol 1987;22:173.

18 Mouradian MM, Juncos JL, Fabbrini G, Chase TN. Motor fluctuations in Parkinson's disease: pathogenetic and therapeutic studies. Ann Neurol 1987;22: 475-9.

19 Ballard PA. Young onset Parkinson's disease: A neurological and neuropsychological study. Ann Neurol 1987;22:173.

20 Fabbrini G, Juncos JL, Mouradian MM, Serrati C, Chase TN. Levodopa pharmacokinetic mechanism and motor fluctuations in Parkinson's disease. Ann Neurol 1987;21:370-6. 